\title{
Biologia populacional de Cyphocarax modestus (Osteichthyes, Curimatidae) no córrego Ribeirão Claro, município de Rio Claro (SP)
}

\author{
Alberto Luciano Carmassi ${ }^{1,2}$; André Teixeira da Silva ${ }^{1}$; \\ Giulianna Rodrigues Rondineli' ${ }^{\text {; Francisco Manoel de Souza Braga }}{ }^{1}$ \\ ${ }^{1}$ Departamento de Zoologia, Instituto de Biociências, Universidade Estadual Paulista - UNESP, \\ Av. 24a, no1515, Bela Vista, CP 199, CEP 13506-900, Rio Claro, SP, Brasil \\ ${ }^{2}$ Autor para correspondência: Alberto Luciano Carmassi, e-mail: carmassi@rc.unesp.br, http://www.rc.unesp.br
}

Carmassi, A. L.; Silva, A. T.; Rondineli, G. R.; Braga, F. M. S. Population biology of Cyphocarax modestus (Osteichthyes, Curimatidae) in the Ribeirão Claro stream, Rio Claro (SP). Biota Neotrop., vol. 8, no. 1, Jan./Mar. 2008. Available from: <http://www.biotaneotropica.org.br/v8n1/en/abstract?article+bn02308012008>.

\begin{abstract}
This work aimed a better understanding of the annual cycle of Cyphocarax modestus in the reservoir of water captation of Ribeirão Claro stream. The growth parameters were estimated through the analysis of length distribution and the relationships among time of smaller growth, alimentary activity, fat accumulation and reproductive period were considered. Besides, the instantaneous rate of natural mortality was calculated. Monthly samplings were accomplished in the Ribeirão Claro stream, in the reservoir of water captation of Rio Claro city. For that, $50 \mathrm{~m}$ of wait net was used, with meshes of 1.5, 2.0, 2.5, 3.0 and $3.5 \mathrm{~cm}$ measured between adjacent knots. The ELEFAN I program was used to estimate the growth parameters, and its application was done using the FISAT program. It was also used the seasonal version of von Bertalanffy's growth curve. It was considered that the reproduction of C. modestus is annual and concentrated from December to February, allowing the identification of different modas in the distributions, an essential condition for the conduction of that analysis type. The estimated parameters were: $\mathrm{K}=0.34$ /year, $\mathrm{L} \infty=15.40 \mathrm{~cm}, \mathrm{C}=0.2, \mathrm{Wp}=0.6$ and $\mathrm{M}=0.949 /$ year, with the identification of four cohorts. The physiologic sequence in the annual cycle of the specie could be noted when data of accumulated fat in the visceral cavity, alimentary activity, reproduction time and time of smaller growth were analyzed together. It was noted that with the beginning of the maturation of gonads, the energy resources stopped being invested in the growth and passed to be used for reproduction.
\end{abstract}

Kyewords: Sagüiru, Corumbataí river sub-basin, reservoir, growth, mortality.

Carmassi, A. L.; Silva, A. T.; Rondineli, G. R.; Braga, F. M. S. Biologia populacional de Cyphocarax modestus no córrego Ribeirão Claro, município de Rio Claro (SP). Biota Neotrop., vol. 8, no. 1, Jan./Mar. 2008. Disponível em: <http://www.biotaneotropica.org.br/v8n1/pt/abstract?article+bn02308012008>.

Resumo: Neste trabalho buscou-se um melhor entendimento do ciclo anual de Cyphocarax modestus na área do reservatório de captação de água do córrego Ribeirão Claro. Os parâmetros de crescimento foram estimados através da análise de distribuição de freqüência de comprimento e as relações entre época de menor crescimento, atividade alimentar, acúmulo de gordura e período reprodutivo foram consideradas. Além disso, a taxa instantânea de mortalidade natural foi calculada. Amostragens mensais foram realizadas no córrego Ribeirão Claro, no reservatório de captação de água do município de Rio Claro. Para isso, $50 \mathrm{~m}$ de rede de espera foram utilizados, com malhas de 1,$5 ; 2,0 ; 2,5 ; 3,0$ e 3,5 cm entre nós adjacentes. O método utilizado para a estimativa dos parâmetros de crescimento foi o ELEFAN I e sua aplicação foi feita utilizando-se o programa FISAT, sendo utilizada a versão sazonal da curva de crescimento de von Bertalanffy. Considerou-se que a reprodução de $C$. modestus é anual e concentrada de dezembro a fevereiro, permitindo assim a identificação de diferentes modas nas distribuições, condição essencial para que esse tipo de análise seja conduzida. Os parâmetros estimados foram: $\mathrm{K}=0,34 / \mathrm{ano}, \mathrm{L} \infty=15,40 \mathrm{~cm}$, $\mathrm{C}=0,2, \mathrm{Wp}=0,6$ e M = 0,949/ano, com a identificação de quatro coortes. Analisando-se conjuntamente os dados de gordura acumulada na cavidade visceral, índice de repleção estomacal, época reprodutiva e de menor crescimento uma sequiência fisiológica no ciclo anual da espécie pôde ser notada: com o início da maturação das gônadas, os recursos energéticos deixaram de ser investidos no crescimento e passaram a ser utilizados na reprodução.

Palavras-chave: Sagüiru, sub-bacia do rio Corumbataí, reservatório, crescimento, mortalidade. 


\section{Introdução}

Estudos envolvendo a determinação da idade e taxa de crescimento podem fornecer informações básicas sobre a estratégia de vida, estrutura de populações e mudanças no crescimento, devido às perturbações ambientais ou à pesca, o que aumenta a compreensão da biologia dos peixes e forma a base dos modelos de dinâmica de populações (Radtke \& Houringan 1990).

O crescimento dos peixes é muito flexível, pois uma mesma espécie pode mostrar padrões diferentes de crescimento em ambientes distintos, como também alcançar a maturidade sexual em diferentes tamanhos ou diferentes idades. Em temperaturas elevadas, típicas de regiões tropicais, as taxas de crescimento são mais altas, o peixe matura com menor idade e o período de vida é mais curto quando comparado a regiões temperadas (Lowe-McConnell 1999). Acretitase que a disponibilidade de alimento seja o principal fator limitante do crescimento em águas tropicais, ao passo que a temperatura o principal fator em águas temperadas (Boujard et al. 1991).

Wootton (1990) comenta que, considerando as condições ambientais como adequadas, o crescimento dos peixes é contínuo ao longo de toda a vida, sendo que o incremento no crescimento decresce com a idade.

Para se estimar a taxa de crescimento, os grupos etários precisam ser determinados, o que não é tarefa fácil em peixes tropicais, visto que as marcas que definem os anéis de crescimento não são muito visíveis (Lowe-McConnell 1999, Boujard et al. 1991). A validade dos estudos sobre idade e crescimento em peixes através das marcas de aposição em estruturas ósseas tem sido muito discutida nos últimos anos em regiões tropicais (Santos \& Barbieri 1993).

Informações sobre o comprimento médio em cada grupo etário, obtidas junto aos dados de freqüência de comprimento, juntamente com a posição das modas associadas com as coortes podem ser utilizados para se obter uma curva de crescimento (Basson et al. 1988).

A família Curimatidae tem distribuição geográfica restrita à América do Sul e sudeste da América Central, com representantes em diferentes ambientes aquáticos (Nelson 1994). Popularmente conhecidos como sagüirus (Britski 1972), são animais de pequeno porte, não possuem dentes e vivem agrupados próximos ao fundo (Fink \& Fink 1978); são abundantes e com importância ecológica nas comunidades de peixes neotropicais por apresentarem hábito alimentar detritívoro (Giora \& Fialho 2003).

Cyphocarax modestus (Fernández-Yépez 1948) é considerada uma espécie forrageira (Godoy 1975), com ciclo reprodutivo anual, com maior intensidade durante o verão (dezembro-janeiro), sendo o tipo de desova parcelada (Barbieri 1995a). Barbieri (1995b) analisando a estrutura populacional e crescimento de $C$. modestus na Represa do Lobo (São Carlos, SP) encontrou cinco anéis etários em suas escamas, sendo estes formados durante o período reprodutivo. Dentre as espécies capturadas por Santos (2005) no Ribeirão Claro, C. modestus esteve entre as mais abundantes.

Neste trabalho buscou-se um melhor entendimento do ciclo anual de Cyphocarax modestus na área do reservatório de captação de água do córrego Ribeirão Claro. Os parâmetros de crescimento foram estimados através da análise de distribuição de frequiência de comprimento e a época de menor crescimento, atividade alimentar, acúmulo de gordura e período reprodutivo foram relacionados. Além disso, a taxa instantânea de mortalidade natural foi calculada.

\section{Material e Métodos}

O presente estudo foi realizado no reservatório de captação de água do córrego Ribeirão Claro (22 $24^{\prime}$ ' 43" S e $47^{\circ} 32^{\prime} 27^{\prime \prime}$ W) localizado nas imediações da Floresta Estadual Navarro de Andrade, no município de Rio Claro.

O reservatório foi formado há mais de 40 anos, com a construção de uma pequena barragem no Ribeirão Claro. A área alagada estende- se por campos de mata secundária e remanescentes de talhões de eucaliptos (Eucaliptus sp.). Atualmente, esse reservatório é responsável pelo abastecimento de $50 \%$ da demanda de água para consumo humano da cidade de Rio Claro (Santos 2005).

O Ribeirão Claro é um dos principais rios da sub-bacia do rio Corumbataí, tendo uma área de drenagem de $270 \mathrm{~km}^{2}$. Das nascentes até seu trecho médio, onde se encontra a estação de captação de água, possui mata ciliar relativamente preservada, após o reservatório, esse córrego recebe despejo de esgoto "in natura" da cidade de Rio Claro, percorrendo trechos tomados por plantações de cana-de-açúcar até sua desembocadura, no rio Corumbataí.

O clima na região é do tipo Cwa de KÖPPEN (Cunha 2000), sendo identificados dois períodos distintos, um com altas temperaturas e alta pluviosidade no período de novembro a abril e outro período com valores baixos de temperatura e pluviosidade, compreendendo os meses de maio a outubro. As temperaturas médias anuais variam entre 20 e $23,7^{\circ} \mathrm{C}$ no mês de janeiro e de 14,9 a $17,1^{\circ} \mathrm{C}$ no mês de julho (Atlas 2004).

As amostragens ocorreram ao longo de um ano, com coletas mensais (de abril de 2003 a março de 2004) no reservatório de captação de água do Ribeirão Claro. Para a coleta dos exemplares, foram utilizados 50 metros de rede de espera com malhas de 1,5; 2,0; 2,5; 3,0 e 3,5 cm entre nós adjacentes, sendo colocadas às 12 horas de um dia e retiradas após 24 horas.

Os exemplares capturados em cada uma das coletas foram colocados em sacos plásticos e mantidos em gelo até serem analisados em laboratório. De cada exemplar foram tomados os seguintes dados: comprimento total e padrão em centímetros, peso total em gramas, grau de repleção do estômago (GR), grau de gordura acumulada na cavidade visceral (GA), sexo e estádio de maturação gonadal (Braga 1990).

Ao grau de repleção estomacal (estado de enchimento do estômago) o valor 1 foi atribuído para estômagos considerados vazios, 2 para estômagos parcialmente cheios e 3 para estômagos completamente cheios. Para o grau de gordura acumulada na cavidade visceral o valor 1 foi dado para a cavidade visceral que não apresentou gordura, 2 para a cavidade visceral parcialmente preenchida com gordura e 3 para a cavidade visceral repleta de gordura. Com relação ao estádio de maturação gonadal foram atribuídas quatro categorias (conforme escala previamente estabelecida): A para gônadas consideradas imaturas, B para gônadas em maturação ou em repouso, C para gônadas maduras e D para aquelas consideradas esgotadas (Braga 1990).

$\mathrm{O}$ índice de repleção estomacal (IR) foi calculado para cada indivíduo de acordo com a expressão IR=PE*100/PT, sendo PE o peso total do conteúdo estomacal e PT o peso total do peixe (Hyslop 1980). Os valores médios mensais do IR foram comparados utilizando-se o teste de Kruskal-Walis (Sokal \& Rohlf 1995) sendo 0,05 o nível de significância.

A relação gonadossomática (RGS), que expressa a porcentagem que as gônadas representam do peso total dos indivíduos, foi calculada mensalmente para todas as fêmeas. De acordo com Vazzoler (1996), a RGS é um indicador eficiente do estado funcional dos ovários. Assim, através das frequiências de fêmeas com gônadas maduras (estádio C) e a variação temporal na RGS, inferências sobre o período reprodutivo da espécie puderam ser feitas.

O método utilizado para a estimativa dos parâmetros de crescimento foi o ELEFAN I (Eletronic Lengths - Frequency Analysis) e a sua aplicação foi feita utilizando-se o programa FISAT (FAO - ICLARM Stock Assessment Tools) (Gayanilo et al. 1996, Gayanylo \& Pauly 1997). Esse programa se baseia no deslocamento modal de sequiências temporais de amostras de comprimento (Castro et al. 2002), utilizando-se a versão sazonal da curva de crescimento de von Bertalanffy (Sparre \& Venema 1997), cujo modelo é $\mathrm{L}_{\mathrm{t}}=\mathrm{L} \infty *\left[1-\exp \left\{\left(-\mathrm{K}^{*}\left(\mathrm{t}-\mathrm{t}_{0}\right)-(\mathrm{CK} / 2 \pi)^{*} \operatorname{seno}\left(2 \pi *\left(\mathrm{t}-\mathrm{t}_{\mathrm{s}}\right)\right)\right\}\right]\right.$, onde $\mathrm{L}_{\mathrm{t}}$ é o comprimento na idade $t, \pi$ equivale a 3,14159 e $t_{\mathrm{s}}$ é a época do ano 
de maior taxa de crescimento, podendo ser calculado através da expressão $t_{\mathrm{s}}=0,5+\mathrm{Wp}$. Assim, o modelo utilizado considera, além dos parâmetros Lo (comprimento assintótico) e K (taxa de crescimento), outros dois parâmetros: Wp ("winter point") e C (amplitude de oscilação sazonal). $\mathrm{O}$ valor de $\mathrm{C}$ está relacionado à diferença de temperaturas superficiais médias da água de inverno e verão; e Wp representa a época do ano em que o crescimento é mais lento, sendo uma relação do número de dias até o mês de menor crescimento da espécie com o número de dias do ano (Pauly \& Gaschutz 1979).

Os pressupostos para a utilização deste método, segundo Pauly \& David (1981), são que: as amostras devem ser representativas da estrutura da população, as estimativas dos parâmetros de crescimento devem ser os mesmos nos diferentes anos, o crescimento deve seguir o modelo de von Bertalanffy e todos os peixes da amostra devem ter o mesmo comprimento na mesma idade. Isaac (1990) acrescenta ainda que o recrutamento deve ocorrer em pulsos.

Apesar do trabalho de Barbieri (1995a) mostrar que a desova de C. modestus na Represa do Lobo é do tipo parcelada, nossos dados sugerem que esta é concentrada em uma única época do ano (de dezembro a fevereiro), fato que não prejudica a utilização do método.

A taxa instantânea de mortalidade natural (M) foi estimada utilizando-se os parâmetros taxa de crescimento (K), comprimento assintótico $(\mathrm{L} \infty)$ e temperatura média da água em graus centígrados no período, segundo a fórmula empírica proposta por Pauly (1980), sendo: $\ln \mathrm{M}=-0,0152-0,279 \ln \mathrm{L} \infty+0,6543 \ln \mathrm{K}+0,463 \ln \mathrm{T}^{\circ}$.

\section{Resultados}

Durante o período de amostragem, foram capturados 370 indivíduos, 170 fêmeas e 170 machos, sendo os demais exemplares imaturos. O menor exemplar capturado apresentou $7,2 \mathrm{~cm}$ de comprimento e o maior 13,6 cm. As maiores capturas ocorreram nos meses maio e julho, com 66 e 72 indivíduos respectivamente (Figura 1).

As estimativas dos parâmetros de crescimento, realizadas a partir dos dados de frequiência de comprimento, foram: $K=0,34 /$ ano e $\mathrm{L} \infty=15,40 \mathrm{~cm}$. O estimador do parâmetro de oscilação $(\mathrm{C})$ foi de 0,2 e o ponto de inverno (Wp) 0,6 , o que indica uma pequena oscilação na temperatura média da água no período e que o crescimento foi menor a partir de julho.

De acordo com Pauly (1998), quando o estoque não é explorado, deve-se considerar a taxa instantânea de mortalidade total $(\mathrm{Z})$ igual à taxa instantânea de mortalidade natural (M). C. modestus não é explorado na área de estudo, pois o acesso ao reservatório não é permitido, além disso, trata-se de uma espécie sem interesse comercial nem de subsistência. Considerando que a temperatura média da água no período foi de $22{ }^{\circ} \mathrm{C}$, encontrou-se $\mathrm{M}=0,949$ /ano.

Através da análise gráfica da frequiência mensal de fêmeas maduras e da variação da RGS (Figura 2), observa-se que o início do desenvolvimento das gônadas ocorre no mês de setembro, sendo o pico de indivíduos maduros no mês de fevereiro. A desova provavelmente ocorre durante os meses de dezembro, janeiro e fevereiro, pois até então os indivíduos estavam apenas desenvolvendo suas gônadas para a reprodução. A partir de fevereiro, com o fim da desova, as fêmeas permanecem em repouso até o mês de agosto, quando novamente inicia-se o processo de maturação gonadal.

Ao se analisar os valores médios do IR (Figura 3), tem-se que os indivíduos se alimentaram durante todo o período, havendo diferença significativa $(\mathrm{p}<0,001)$ entre os meses considerados. Os meses de maio e junho obtiveram os maiores valores de IR, sugerindo uma maior atividade alimentar.

Com relação à gordura acumulada na cavidade visceral, durante os meses de abril a agosto foram observadas as maiores freqüências de indivíduos com grau máximo de gordura, e de setembro a março as menores freqüências (Figura 4).

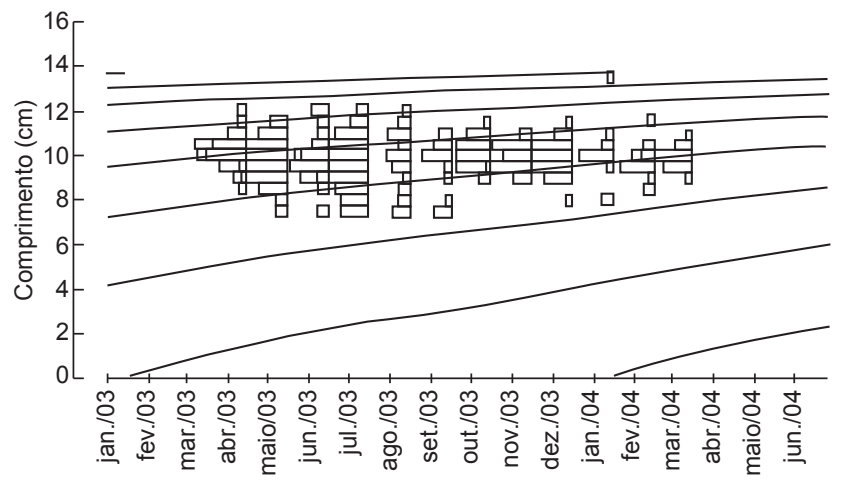

Figura 1. Curva de crescimento de Cyphocarax modestus reestruturada para freqüência mensal de comprimento total, calculada por meio do sistema ELEFAN I (pacote computacional FISAT).

Figure 1. Curves of growth of Cyphocarax modestus restructured for monthly frequency of total length, made calculations through the system ELEFAN I (package computacional FISAT).

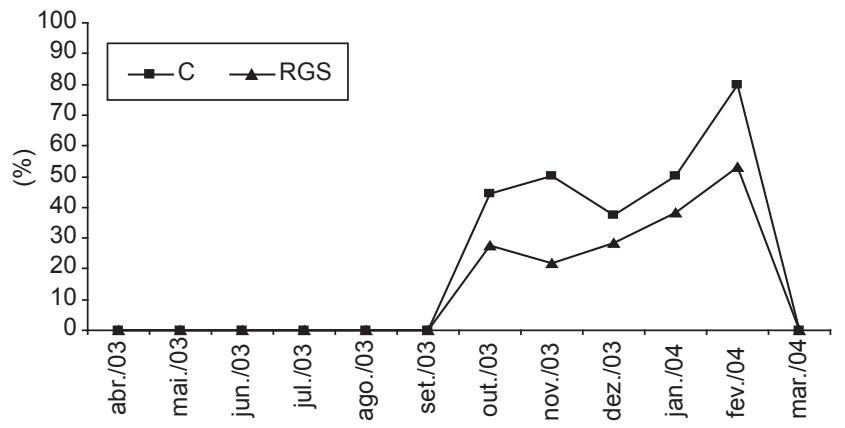

Figura 2. Variação mensal na porcentagem de fêmeas maduras $(C)$ e na relação gonadossomática (RGS) durante o período de amostragem (abril/03 a março/04) no reservatório de captação de água do córrego Ribeirão Claro.

Figure 2. Monthly variation in the percentage of mature females $(\mathrm{C})$ and in the relationship gonadosomaticc (RGS) during the sampling period (april/03 to march/04) in the reservoir of water capitation of Ribeirão Claro stream.

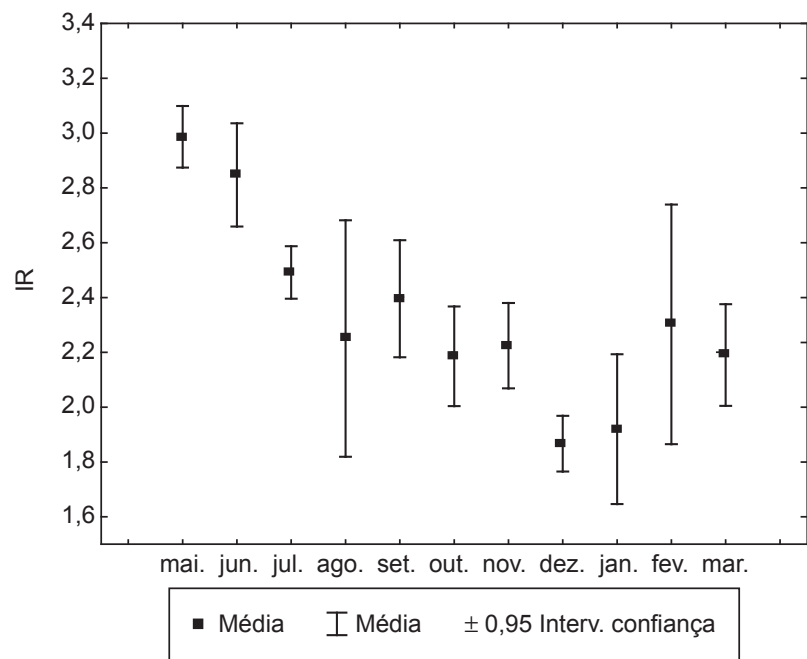

Figura 3. Valores médios mensais do Índice de Repleção (IR) para os indivíduos de Cyphocarax modestus capturados durante o período de amostragem no reservatório de captação de água do córrego Ribeirão Claro.

Figure 3. Monthly medium values of the Index of Repletion (IR) for the individuals of Cyphocarax modestus captured during the sampling period in the reservoir of water capitation of Ribeirão Claro stream. 


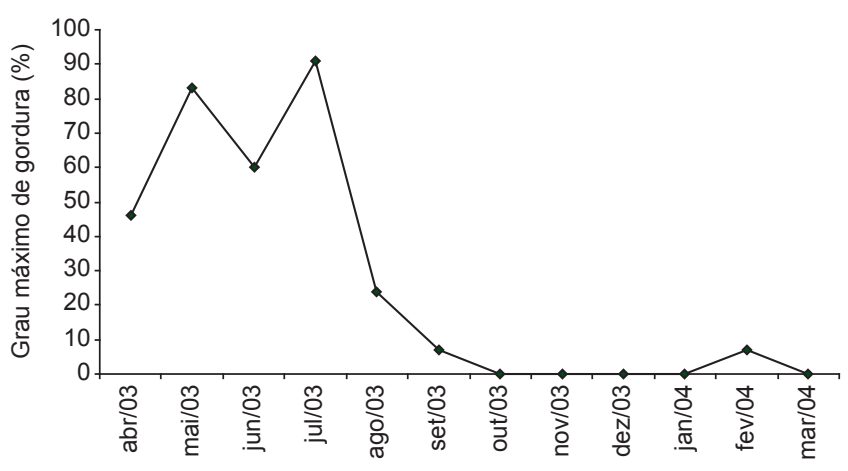

Figura 4. Variação mensal do grau máximo de gordura na cavidade visceral para os indivíduos de Cyphocarax modestus capturados durante o período de amostragem (abril/03 a março/04) no reservatório de captação de água do córrego Ribeirão Claro.

Figure 4. Monthly variation of the maximum degree of accumulated fat in the visceral cavity for the individuals of Cyphocarax modestus captured during the sampling period (april/03 to march/04) in the reservoir of water capitation of Ribeirão Claro stream.

\section{Discussão}

Os parâmetros de crescimento são geralmente obtidos relacionando-se a idade com um tamanho em um modelo de crescimento. A equação de crescimento mais utilizada para predizer o crescimento em peixes é a de von Bertalanffy (Mateus 2003), que por se apoiar em conceitos fisiológicos, fornece uma representação satisfatória do crescimento do peixe (Beverton \& Holt 1957).

Segundo Beverton \& Holt (1957) os valores assintóticos podem ser influenciados pelo suprimento alimentar e densidade populacional, enquanto que a taxa de crescimento é genética e fisiologicamente determinada, embora esses autores reconheçam que existe uma interação matemática entre os parâmetros envolvidos.

Em águas temperadas, tais parâmetros podem ser obtidos através da contagem de anéis anuais em partes duras, como escamas e otólitos. Esses anéis são formados devido às fortes flutuações ambientais entre o verão e o inverno. Em áreas tropicais não ocorrem essas flutuações marcantes, sendo rara a formação destes anéis, tornando difícil a determinação da idade através deste método. No entanto, existem diversos trabalhos que fizeram uso de métodos diretos e obtiveram êxito, dentre eles Nomura \& Taveira (1978), Barbieri (1992), Barbieri (1995b), Ambrósio \& Hayashi (1997) e Braga (1999).

Como as distribuições de frequiência de comprimento são muito mais fáceis de serem obtidas em populações biológicas do que distribuições de idade, uma curva de crescimento pode ser traçada a partir das informações sobre comprimento médio na idade pela posição das modas associadas com as coortes (Basson et al. 1988).

No presente estudo, os parâmetros de crescimento foram estimados utilizando-se um método indireto, considerando que a reprodução de Cyphocarax modestus é anual e concentrada de dezembro a fevereiro, permitindo assim a identificação de diferentes modas nas distribuições, condição essencial para que esse tipo de análise seja conduzida (Sparre \& Venema 1997). Quando esses pulsos não são claramente identificáveis na atividade reprodutiva, a estimativa dos parâmetros de crescimento a partir das distribuições de frequiência pode ser problemática (Basson et al. 1988).

O método utilizado nesse estudo vem sendo aplicado para estimativa dos parâmetros de crescimento para outras espécies de peixes de pequeno porte. Dentre eles podemos citar Lizama \& Ambrósio (2003) trabalhando com Moenkausia intermedia na planície de inundação do alto rio Paraná, Gomiero et al. (2007) estudando a pirapitinga do sul (Brycon opalinus) no Parque Estadual da Serra do Mar e Braga et al. (2007) para duas espécies de Characidium na microbacia do Ribeirão Grande.

Os resultados por nós encontrados mostraram a existência de quatro coortes na população de Cyphocarax modestus no reservatório de água do Ribeirão Claro e que a época de menor crescimento ocorreu no mês de julho.

Barbieri (1995b) encontrou para a mesma espécie em outra localidade através de método de contagem de anéis etários em escamas, cinco anéis, que se formaram no bimestre dezembro-janeiro, período em que esta espécie se encontrava em maior atividade reprodutiva.

Segundo Nikolskii (1963) muitas espécies de peixes podem apresentar nas escamas anéis etários resultantes da interrupção da alimentação e da exaustão, decorrentes do processo reprodutivo. Esses anéis são os de desova e são úteis na estimativa da idade de peixes tropicais de água doce que geralmente não formam anéis de inverno (Braga 1999).

Entre os fatores que influenciam o crescimento, encontram-se os fatores exógenos, como temperatura da água e $\mathrm{pH}$, e os endógenos, dos quais os mais evidentes estão relacionados ao desenvolvimento das gônadas e desova, que por sua vez estão associados a processos fisiológicos (Ambrósio \& Hayashi 1997). Holzbach et al. (2005) atribuíram à disponibilidade de alimento, variabilidade genética e outros fatores ambientais relacionados ao hábitat influência sobre o crescimento de outro curimatídeo, Steindachnerina insculpita.

Nomura \& Hayashi (1980) estudando os caracteres merísticos de uma outra espécie de sagüiru, Curimatus gilberti, mostraram através da leitura de vértebras e ajuste da curva de crescimento de von Bertalanffy, que o comprimento teórico máximo das fêmeas foi de 155,2 mm e o dos machos 151,9 mm. Para Cyphocarax modestus o comprimento assintótico encontrado foi de $15,40 \mathrm{~cm}$. A taxa de crescimento determina o tipo de crescimento apresentado por uma espécie e é geralmente inversa ao valor do comprimento assintótico e à logevidade (Sparre et al. 1989, King 1996).

Vários são os fatores que agem durante o ciclo de vida dos peixes e que atuam na mortalidade natural, desde a fase de ovo até a morte pela senilidade. Diferenças interespecíficas nas taxas de mortalidade refletem adaptações das espécies ao ambiente em que vivem (Gomiero et al. 2007). A alta taxa de mortalidade de Cyphocarax modestus pode estar relacionada com a intensa predação que sofre no local de estudo, uma vez que serve de alimento para outras espécies como Acestrorhyncus lacustris, o qual alimenta-se principalmente de C. modestus (Silva 2005).

A maturação das gônadas e/ou atividades reprodutivas implicam na utilização de recursos obtidos a partir do alimento ingerido e, principalmente, de reservas energéticas depositadas em diferentes partes do organismo (Agostinho et al. 1990).

Godinho (1994) afirma que o desenvolvimento de gordura tem ligação com a reprodução. A mobilização de reservas energéticas durante o desenvolvimento das gônadas foi constada por Barbieri \& Verani (1987) para Hypostomus aff. plecostomus; Agostinho et al. (1990) para Rhinelepis aspera; Tavares \& Godinho (1994) para Leporinius piau; Bennemann et al. (1996) para Schizodon intermedius, Pimelodus maculatus, Acestrorhynchus lacustris e Steindachnerina insculpita; Brito et al. (1999) para Leporinus friderici, entre outros.

Assim como para as espécies citadas acima, o início do processo de amadurecimento das gônadas de $C$. modestus coincidiu com o consumo das reservas de gordura. Com a redução dessas reservas, ocorreu um aumento na atividade alimentar, provavelmente para suprir as necessidades nutricionais do animal e também para a reposição das reservas consumidas.

A época de maior atividade alimentar ocorreu nos meses de maio e junho, coincidindo com o período de baixa incidência de chuvas, 
que proporcionou maior transparência da água, possibilitando a proliferação das algas, principalmente aquelas associadas ao substrato, aumentando a disponibilidade desse tipo de alimento para essa espécie. A maior captura nos meses de maio e julho pode indicar que a espécie encontrava-se mais ativa.

Barbieri et al. (1982), Hartz et al. (1996) e Giora \& Fialho (2003) constataram maiores freqüências de estômagos repletos antes e após o período reprodutivo, não havendo interrupção da atividade alimentar durante a reprodução como o observado por nós para $C$. modestus.

Quando os dados de gordura acumulada na cavidade visceral, índice de repleção estomacal, época reprodutiva e de menor crescimento foram analisados conjuntamente, a existência de uma seqüência fisiológica no ciclo anual da espécie pôde ser notada.

Diante disso, pode-se supor que a partir do momento em que as gônadas começam a maturar, os recursos energéticos deixam de ser investidos no crescimento e passam a ser utilizados para esse fim. $\mathrm{O}$ que foi indicado na seqüência de maior atividade alimentar, armazenamento de gordura e ocorrência de reprodução.

\section{Agradecimentos}

À FAPESP pela bolsa de iniciação científica concedida ao primeiro autor (proc. $n^{\circ} 03 / 02020-7$ ).

\section{Referências Bibliográficas}

AGOSTINHO, A.A., BARBIERI, G., VERANI, J.R. \& HAHN, N.S. 1990. Variação do fator de condição e do índice hepatossomático e suas relações com o ciclo reprodutivo em Rhinelepis aspera (Agassis, 1829) (Osteichthyes, Loricariidae) no rio Paranapanema, Porecatu, PR. Ciência e Cultura, 42(9):711-714.

AMBRÓSIO, A.M. \& HAYASHI, C. 1997. Idade e crescimento de Steindachnerina insculpita (Fernandes-Yepes 1948), (Characiformes, Curimatidae) da planície de inundação do Alto rio Paraná. Rev. Bras. Biol., 57(4):687-698.

ATLAS ambiental da bacia do rio Corumbataí. Disponível em <http://www. rc.unesp.br/igce/ceapla/atlas>. Acesso em 07.set.2004.

BARBIERI, G. 1992. Biologia de Astyanax scabripinnis paranae (Characiformes, Characidae) do Ribeirão do Fazzari, São Carlos, Estado de São Paulo. I. Estrutura populacional e crescimento. Rev. Bras. Biol., 52(4):579-588

BARBIERI, G. 1995a. Biologia de Cyphocarax modesta (Hensel, 1869) (Characiformes, Curimatidae) da Represa do Lobo, Estado de São Paulo. II. Dinâmica da reprodução e influência de fatores abióticos. B. Inst. Pesca, 22(2):57-62.

BARBIERI, G. 1995b. Biologia de Cyphocarax modesta (Hensel, 1869) (Characiformes, Curimatidae) da Represa do Lobo, Estado de São Paulo. I. Estrutura populacional e crescimento. B. Inst. Pesca, 22(2):49-56.

BARBIERI, G. \& VERANI, J.R. 1987. O fator de condição como indicador do período de desova em Hypostomus aff. plecostomus (Linnaeus, 1758) (Osteichthyes, Loricariidae), na represa do Monjolinho (São Carlos, SP). Ciência e Cultura, 39(7):655-658.

BARBIERI, G., VERANI, J.R. \& BARBIERI, M.C. 1982. Dinâmica qualitativa da nutrição de Hoplias malabaricus (Bloch, 1974) na represa do Lobo (Brotas-Itirapina-SP) (Pisces, Erythrinidae). Rev. Bras. Biol., 42(2):95-302.

BASSON, M., ROSENBERG, A.A. \& BEDDINGTON, J.R. 1988. The accuracy and reliability of two new methods for estimating growth parameters from length-frequency data. J. Cons. Int. Explor. Mer., 44:277-285.

BENNEMANN, S.T., ORSI, M.L. \& SHIBATA, O. 1996. Atividade alimentar de espécies de peixe do rio Tibagi, relacionada com o desenvolvimento de gordura e gônadas. Rev. Bras. Zool., 13(2):501-512.

BEVERTON, R.J.H. \& HOLT, S.J. 1957. On the dynamics of exploited fish populations. Fishery Invest. London, 2(19):1-533.
BOUJARD, Y., LECOMTE, F., RENNO, J.F., MEUNIER, F. \& NEVEU, P. 1991. Growth in four populations of Leporinus friderici (Bloch, 1794) (Anostomidae, Teleostei) in French Guiana. J. Fish Biol., 38:387-397.

BRAGA, F.M.S. 1990. Aspectos da reprodução e alimentação de peixes comuns em um trecho do rio Tocantins entre Imperatriz e Estreito, Estado do Maranhão e Tocantins, Brasil. Rev. Bras. Biol., 50(3):547-558.

BRAGA, F.M.S. 1999. Idade, crescimento e taxas de mortalidade de Astyanax bimaculatus (Characidae, Tetragonopterinae) na represa de Barra Bonita, rio Piracicaba (SP). Naturalia, 24:239-250.

BRAGA, F.M.S; SOUZA, U.P. \& CARMASSI, A.L. 2007. Dinâmica populacional de Characidium lauroi e C. alipioi (Teleostei, Crenuchidae) na microbacia do Ribeirão Grande, serra da Mantiqueira oriental (SP). Acta. Sci. Biol. Sci., 29(3):281-287.

BRITO, M.F.G., SANTOS, G.B. \& BAZZOLI, N. 1999. Reprodução de Leporinus friderici (Pisces: Anostomidae) no reservatório de Itumbiara, GO. Bios, 7(7):33-40.

BRITSKI, H.A. 1972. Peixes de água doce do estado de São Paulo: Sistemática. In: Comissão interestadual da bacia do Paraná-Uruguai, Poluição e piscicultura. São Paulo. p.88-108.

CASTRO, P.M.G., CERGOLE, M.C., CARNEIRO, M.H., MUCINHATO, C.M.D. \& SERVO, G.J.M. 2002. Crescimento, mortalidade e taxa de explotação do goete, Cynoscion jamaicensis (Perciformes: Sciaenidae), na região sudeste/sul do Brasil. B. Inst. Pesca, 28(2):141-153.

CUNHA, M.A.A. 2000. Diagnóstico da qualidade das águas da bacia do Ribeirão Claro (SP) através de diversas análises inclusive multivariadas. Dissertação de Mestrado, Instituto de Ciências Biológicas, Universidade Federal de Minas Gerais, Belo Horizonte.

FINK, W.I. \& FINK, S. 1978. A Amazônia central e seus peixes. Supl. Acta Amazônica, 8(4):19-42.

GAYANILO JR, F.C., SPARRE, P. \& PAULY, D. 1996. The FAO-ICLARM Stock Assessment Tools (FISAT) User's guide. FAO Computerized Information Series (Fisheries), 6:1-186.

GAYANILO JR., F.C. \& PAULY, D. 1997. FAO-ICLARM Stock Assessment Tools (FISAT) Reference Manual. FAO Computerized Information Series (Fisheries), 8:1-262.

GIORA, J. \& FIALHO, C.B. 2003. Biologia alimentar de Steindachnerina brevipinna (Characiformes, Curimatidae) do rio Ibicuí-Mirim, Rio Grande do Sul, Brasil. Iheringia, 93(3):277-281.

GODINHO, H.M. 1994. Biologia reprodutiva da piaba-facão, Triportheus guentheri (Characiformes, Characidae) e o manejo hidrológico da represa de Três Marias. Rev. Bras. Biol., 54(3):515-524.

GODOY, M.P. 1975. Peixes do Brasil. Editora Franciscana, Piracicaba.

GOMIERO, L.M., CARMASSI, A.L. \& BRAGA, F.M.S. 2007. Crescimento e mortalidade de Brycon opalinus (Characiformes, Characidae) no Parque Estadual da Serra do Mar, Mata Atlântica, Estado de São Paulo. Biota Neotropica, 7(1):http://www.biotaneotropica.org.br/v7n1/ pt/abstract?article+bn00207012007 (último acesso em 25/07/2007)

HARTZ, S.M., MARTINS, A. \& BARBIERI, M.C. 1996. Dinâmica da alimentação e dieta de Oligosarcus jenynsii (Gunther, 1862) na lagoa Caconde, Rio Grande do Sul, Brasil (Teleostei, Characidae). B. Inst. Pesca, 23:411-429.

HOLZBACH, A.J., BAUMGARTNER, G., BERGMANN, F., REZENDENETO, L.B., BAUMGARTNER, D., SANCHES, P.V. \& ANDRÉ, E. 2005. Caracterização populacional de Steindachnerina insculpita (Fernández-Yépez, 1948) (Characiformes, Characidae) no rio Piquiri. Acta. Sci. Biol. Sci., 27(4):347-353.

HYSLOP, S.M. 1980. Stomach contents analysis: a review of methods and their appliacation. J. Fish Biol., 17:411-429.

ISAAC, V.J. 1990. The accuaracy of some lenght-based methods for fish population studies. ICLARM. Technical Report, 27:1-81.

KING, M. 1996. Fisheries biology, assessment and management. Black Science, Oxford.

LIZAMA, M.A.P. \& AMBRÓSIO, A.M. 2003. Crescimento, recrutamento e mortalidade do pequi Moenkausia intermedia (Osteichthyes, Characidae) 
na planície de inundação do alto rio Paraná, Brasil. Acta Sci. Biol. Sci., 25(2):329-333.

LOWE-McCONNELL, R.H. 1999. Estudos ecológicos em comunidades de peixes tropicais. Editora USP, São Paulo.

MATEUS, L.A.F. 2003. Ecologia da pesca de quatro grandes bagres (Siluriformes: Pimelodidae) na bacia do rio Cuiabá, pantanal mato-grossense. Tese de Doutorado, Instituto de Biociências - Universidade Estadual Paulista, Rio Claro, São Paulo.

NELSON, J.S. 1994. Fishes of the world. Ed. John Wiley \& Sons, New York.

NIKOLSKII, G.V. 1963. The ecology of fishes. Academic Press, London.

NOMURA H. \& HAYASHI C. 1980. Caracteres merísticos e biologia do sagüiru, Curimatus gilberti (Quoy \& Gaimard, 1824), do rio Morgado (Matão, São Paulo) (Osteichthyes, Curimatidae). Rev. Bras. Biol., 40(1):165-176.

NOMURA, H. \& TAVEIRA, A.C.D. 1978. Biologia do saguiru, Curimatus elegans Steindachner, 1874 do rio Mogi Guaçu, São Paulo (Osteichthyes, Curimatidae). Rev. Bras. Biol., 39(2):331-339.

PAULY, D. 1980. On the interrelationships between natural mortality, growth parameters, and environmental temperature in 175 fish stocks. International Council for the Exploration of the Sea, 39(2):175-192.

PAULY, D. 1998. Tropical fishes: patterns and propensities. J. Fish Biol., 53:1-17.

PAULY, D. \& DAVID, N. 1981. Elefan I, a basic program for the objective extraction of growth parameter from length frequence data. Meeresforsch, 28(4):205-211.

PAULY, D. \& GASCHUTZ, G. 1979. A simple method for fitting oscillating length growth data, with a program for pocket calculator. International Council for the Exploration of the Sea. Council Meeting 1979/G. Demersal Fish Committee, 26p.
RADTKE, R.L. \& HOURIGAN, T.F. 1990. Age and growth of the Antartic fish Nototheniops nudifrons. Fish Bull., 88:557-571.

SANTOS, A.T.B. 2005. Estudo da comunidade de peixes no Ribeirão Claro, Rio Claro, SP. Dissertação de Mestrado, Instituto de Biociências - Universidade Estadual Paulista, Rio Claro, São Paulo.

SANTOS, G.B. \& BARBIERI, G. 1993. Idade e crescimento do "piau gordura", Leporinus piau Fowler, 1941, na represa de Três Marias (Estado de Minas Gerais) (Pisces, Ostariophyso, Anostomidae). Rev. Bras. Biol., 53(4):649-658.

SILVA, A.T. 2005. Dieta e dinâmica da nutrição do peixe-cachorro, Acestrorhynchus lacustris (Reinhardt, 1874) (Characiformes, Acestrorhynchinae), no ponto de captação de água do Ribeirão Claro. Trabalho de Formatura, Instituto de Biociências - Universidade Estadual Paulista, Rio Claro, São Paulo.

SOKAL, R.R. \& ROHLF, F.J. 1995. Biometry: the principles of statistics in biological research. WH Freeman and Company, New York, 887p.

SPARRE, P. \& VENEMA, S.C. 1997. Introdução à avaliação de mananciais de peixes tropicais. Parte 1: Manual. FAO, Roma.

SPARRE, R.R.; URSIN, E. \& VENEMA, S.C. 1989. Introduction to tropical fish stock assessment. Parte 1: Manual. FAO, Roma.

TAVARES, E.F. \& GODINHO, H.P. 1994. Ciclo reprodutivo do peixe piaugordura (Leporinus piau Fowler, 1941) da represa de Três Marias, rio São Francisco. Revista Ceres, 41(233):28-35.

VAZZOLER, A. E. A. de M. 1996. Biologia da reprodução de peixes teleósteos: teoria e prática. EDUEM, Maringá; SBI, S. Paulo, 169 p.

WOOTTON, R. J. 1990. Ecology of teleost fishes. Chapman \& Hall, London.

Recebido em $27 / 08 / 07$

Versão reformulada recebida em 22/01/08

Publicado em 18/02/08 